\title{
Acoustic Signal Analysis by Teager-Huang Transform for Milling Chatter Recognition
}

\author{
Rong-Mao Lee, ${ }^{1 *}$ Yi-Hsing Yu, ${ }^{2}$ Cheng-Chi Wang, ${ }^{3}$ \\ Tsung-Chia Chen, ${ }^{2}$ and Pongpon Nilaphruek ${ }^{4}$ \\ ${ }^{1}$ Computer and Intelligent Robot Program for Bachelor Degree, National Pingtung University, \\ No. 4-18, Minsheng Rd., Pingtung City, Pingtung County 90003, Taiwan \\ ${ }^{2}$ Department of Mechanical Engineering, National Chin-Yi University of Technology, \\ No. 57, Sec. 2, Zhongshan Rd., Taiping Dist., Taichung City 41170, Taiwan \\ ${ }^{3} \mathrm{Ph}$. D. Program, Graduate Institute of Precision Manufacturing, National Chin-Yi University of Technology, \\ No. 57, Sec. 2, Zhongshan Rd., Taiping Dist., Taichung City 41170, Taiwan \\ ${ }^{4}$ Department of Mathematics and Computer Science, Rajamangala University of Technology Thanyaburi, \\ 39 Moo 1, Klong6, Khlong Luang Pathum Thani 12110, Thailand
}

(Received February 3, 2019; accepted December 23, 2019)

Keywords: milling vibration, milling noise, time-frequency domain, Teager-Huang transform

Many types of sensor have been utilized to monitor milling vibration, and many analysis methods are devoted to the investigation of milling vibration or milling dynamics. In this work, a noncontact sensor and a time-frequency domain analysis method were applied to identify the state of milling vibration. A microphone was employed in practical tests to record the milling dynamics. The Teager-Huang transform (THT) was adopted for the acoustic signal analysis owing to its high resolution in the time-frequency domain. The potential frequency range for the analysis of milling dynamics is reported in this work to improve the recognition accuracy of milling vibration limited by the effect of environmental noise. The THT was used to distinguish the chatter state from the normal milling dynamics. In addition, the statistical index called the coefficient of variation was applied to define the threshold of chatter occurrence. Milling experiments (including dry and wet cuttings) were performed to verify the proposed chatter detection method.

\section{Introduction}

The recognition of milling chatter should be discussed in two aspects: the disposed sensor and the signal processing method. Dynamometers, accelerometers, and acoustic sensors are the main data sources against milling dynamics up to the present. ${ }^{(1)}$ There are two types of acoustic sensor, namely, the acoustic emission (AE) sensor and the microphone, which can be applied to record variations in the milling process. The main measurement frequency ranges of these four types of sensor are very different. The signal frequencies measured by dynamometers and accelerometers are usually below $5 \mathrm{kHz}$ (low frequency). For microphones and AE sensors, the measured signal frequencies can be $0-80 \mathrm{kHz}$ and beyond $400 \mathrm{kHz}$, respectively. These *Corresponding author: e-mail: maxmou@mail.nptu.edu.tw https://doi.org/10.18494/SAM.2020.2671 
acoustic sensors are more sensitive to the energy variations inside the milling dynamics than the dynamometers and accelerometers. For example, the efficacy of cutter sharpness (tool wear) detection by the AE sensor was preliminarily validated. ${ }^{(2-5)}$ However, the competence to detect milling chatter using AE signals is still indefinite owing to the significant signal strength decay in the high frequency range. As a result, a microphone was employed in this work owing to its high sensitivity against the milling dynamics, its sufficient signal bandwidth, and its passable signal strength decay.

The second part of the milling chatter identification is on the method of sensor signal processing. Grossi et al. introduced an experimental approach to evaluate the optimal stable cutting parameters. ${ }^{(6)}$ The approach named spindle speed ramp-up (SSR) was applied to the tests, and the chatter occurrence was detected by the order analysis technique. Only a few tests have to be performed and the entire stability lobe diagram (SLD) can be obtained. Lamraoui et al. proposed a chatter detection methodology by neural network classification and feature selection. ${ }^{(7)}$ Vibration signals were filtered by a multiband resonance filter to increase the signal-to-noise $(\mathrm{S} / \mathrm{N})$ ratio and the sensitivity of generated features. The stability of selected features was finally determined by two neural network approaches. A real-time chatter monitoring and suppression method was performed by Han et al. ${ }^{(8)}$ The estimation of signal parameters via the rotational invariance technique (ESPRIT) algorithm was adopted to extract the frequency characteristics of acceleration signals. The cutting state was then categorized on the basis of the amplitude-frequency characteristics of identified acceleration signals with the aid of the hidden Markov model (HMM). A study focused on timely chatter detection was published in 2018. ${ }^{(9)}$ The measured vibration signal was decomposed by the Gabor filter bank, and the Teager nonlinear energy operator was used to track signal variations in instantaneous frequency (IF) and amplitude. A similar study based on the empirical mode decomposition (EMD) was published. ${ }^{(10)}$ The vibratory signals were decomposed by the EMD, and a series of intrinsic mode functions (IMFs) corresponding to instantaneous vibration frequencies were then obtained. By selecting the primary IMFs, the chatter phenomenon was finally judged using the mean square frequency and self-correlation coefficient. There are other methods devoted to the applications of online chatter detection, such as chatter identification based on wavelet transform (WT) and support vector machine (SVM), ${ }^{(11)}$ Hilber-Huang transform (HHT), ${ }^{(12)}$ and short-time Fourier transform (STFT). ${ }^{(13,14)}$

The time-frequency signal processing tools, such as STFT, WT, HHT, and Teager-Huang transform (THT), are gradually applied to dynamic signal analyses owing to their superior performance in both time and frequency domains simultaneously. The signal-resolving powers of STFT and WT are limited by the time window function and the mother wavelet type, respectively. In addition, the capability of signal analysis for the frequency and amplitude of THT is better than that of HHT. ${ }^{(15,16)}$ Consequently, THT was employed in this work to investigate the milling noise.

The purpose of this work is to design an efficient analysis process to detect chatter by milling noise analysis. A microphone was utilized to record the milling dynamics, and the measured acoustic signals were analyzed by THT. Owing to the high sensitivity of the microphone against energy variation in the milling process, a slight variation of milling noise 
during the early stage of chatter occurrence may be observed. The frequency range for signal analysis was determined using the THT results to decouple most of the environmental noise. The statistical index called the coefficient of variation (CV) was used to define the threshold of chatter occurrence on the basis of the THT results. The performance of the proposed method was finally investigated by practical milling tests.

\section{Research Process and Methods}

The research process is shown in Fig. 1. The first stage (Stage A) is to confirm the essential function of the microphone against the milling dynamics. Two accelerators were deployed to be the comparison baseline of chatter detection. Once the THT results of microphone signals are validated to be effective for milling monitoring, the CV of IF will be calculated. The CV threshold for chatter detection was defined on the basis of the CV results of IF. The second stage (Stage B) is to verify the performance of the proposed method. The milling parameters of Stage B are different from those of Stage A. The diagnosis of chatter occurrence by microphone signal analyses will finally be confirmed with the aid of accelerator signals and the SLD of the test rig. Since the cutting fluid was involved in the milling tests, a waterproof device for the microphone ${ }^{(17)}$ was used in this work. The prototype of the waterproof device is shown in Fig. 2.

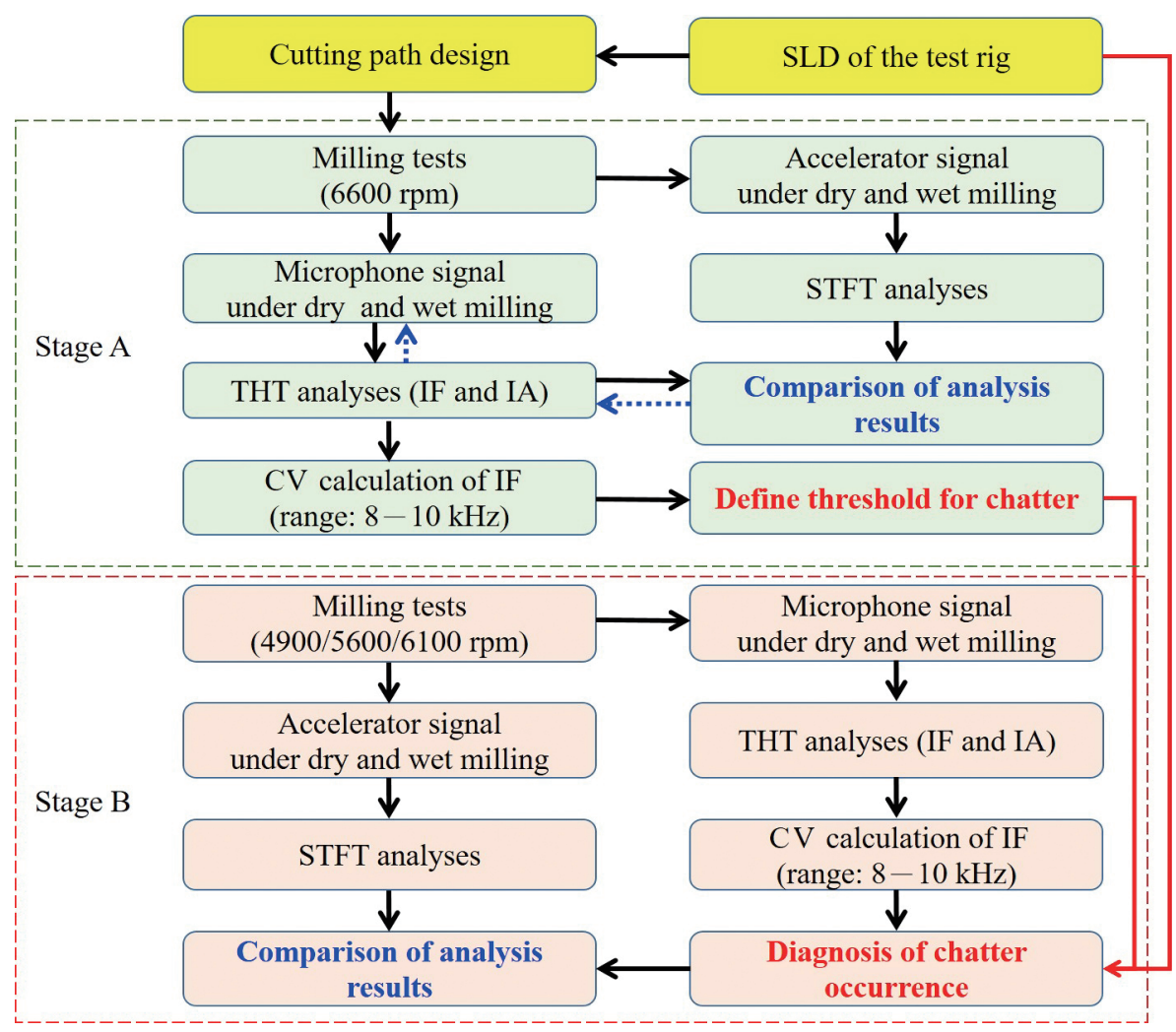

Fig. 1. (Color online) Two-stage research procedure. 


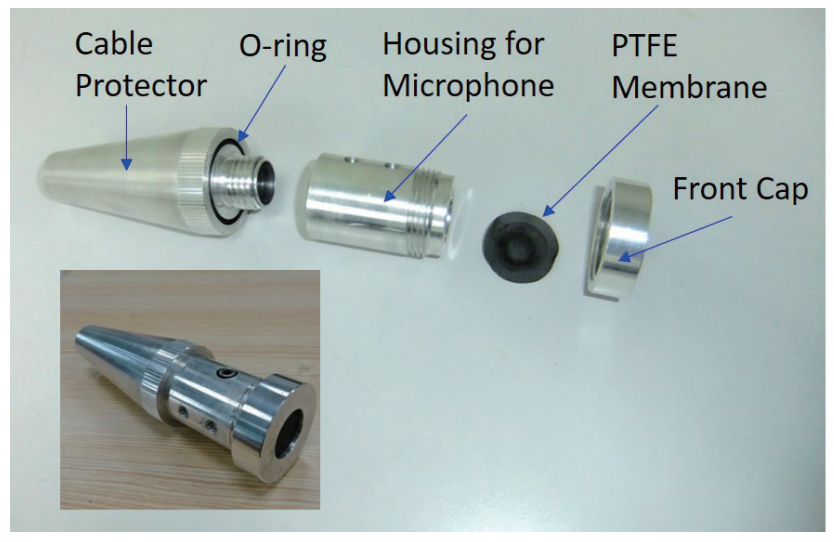

Fig. 2. (Color online) Waterproof device for the microphone. ${ }^{(17)}$

\subsection{THT}

THT is an approach for nonstationary signal analysis. This method is based on the EMD algorithm $^{(18)}$ and the Teager energy operator (TEO). ${ }^{(19)}$ The signal processing architecture of THT is shown in Fig. $3 .^{(15)}$ The raw data is firstly decomposed by the EMD into IMFs. IMFs represent the oscillation modes embedded in the signal, and the IMF in each cycle involves only one oscillation mode. IMFs are then demodulated by the TEO into IF and the instantaneous amplitude (IA). The TEO is the kernel of energy separation algorithm (ESA) demodulation, and it has been successfully applied to speech processing. ${ }^{19)}$ Since the microphone signal is composed of the superposition of AM-FM signals such as the speech signal, THT could be sufficient for the milling noise analysis. Finally, an energy-time-frequency representation of the input signal will be accomplished with the IF and IA results.

\subsection{Selection of frequency range for microphone signal analysis}

The THT results of the environmental noise and milling process are shown in Fig. 4. Most of the environmental noise frequencies are below $7 \mathrm{kHz}$. The frequency ranges of milling dynamics and environmental noise partially overlap, as shown in Fig. 4(b). The frequency range of $8-10 \mathrm{kHz}$ was finally determined for microphone signal analysis to improve the coupling of environmental noise and to avoid the attenuation of the high-frequency signal.

\section{$2.3 \mathrm{CV}$}

Since the frequency range and amplitude of the first IMF $\left(\mathrm{IMF}_{1}\right)$ are the largest among all the IMFs, IMF 1 was used to evaluate the stability of cutting force variation. ${ }^{(12)}$ However, some information within the other IMFs may be lost in the following analysis. In this work, a statistical index called CV was adopted to observe the variation of milling dynamics, which is defined as 


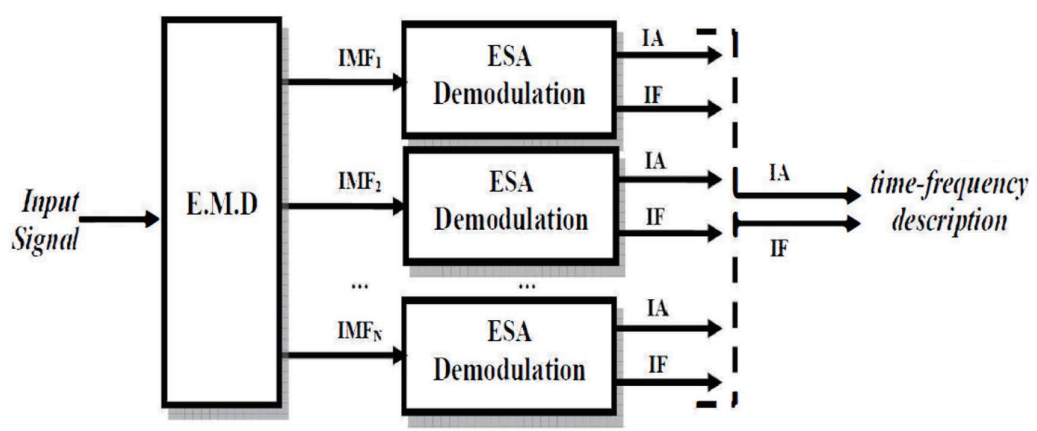

Fig. 3. Signal processing architecture of THT. ${ }^{(15)}$

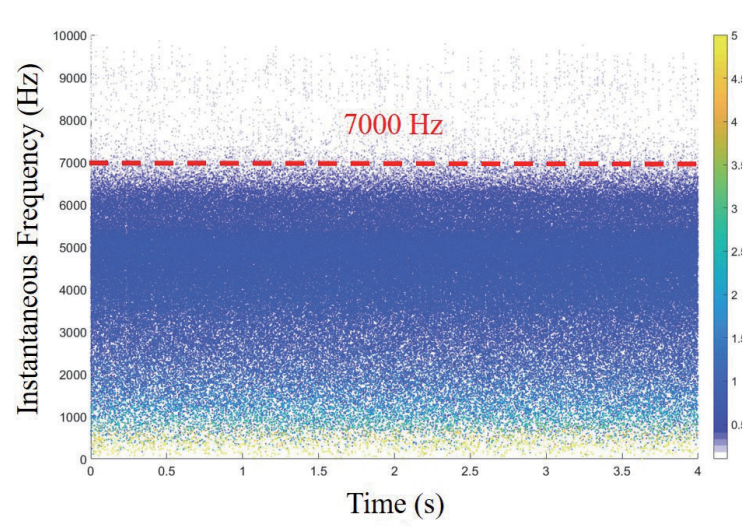

(a)

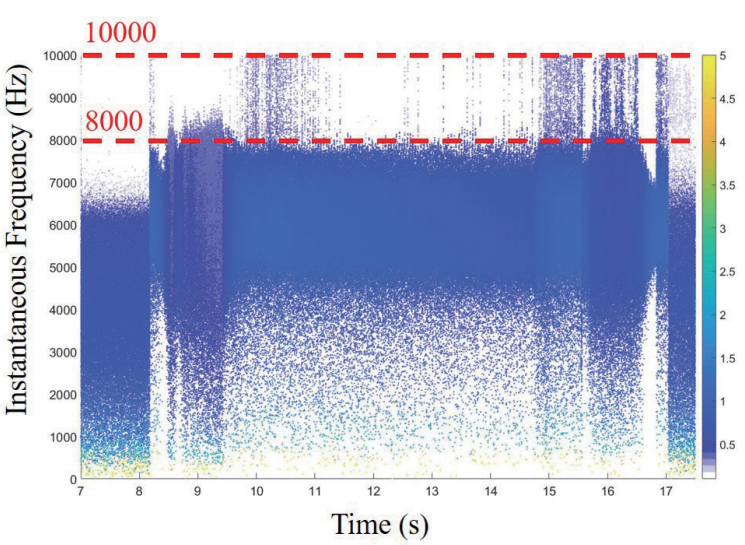

(b)

Fig. 4. (Color online) THT results of (a) environmental noise and (b) milling process.

$$
C V=\frac{\sqrt{\frac{1}{N} \sum_{i=1}^{N}\left(x_{i}-\mu\right)^{2}}}{\mu} \times 100 \%,
$$

where $N$ is the amount of data, $x_{i}$ is IF, and $\mu$ is the average frequency. The data source of the CV calculation is the IF of THT results between 8 and $10 \mathrm{kHz}$. As shown in Fig. 4(b), the dispersion degree of signal frequencies will vary along with the milling dynamics. Therefore, the threshold for chatter detection can be defined using the CV.

\subsection{Experimental design}

The test rig is shown in Fig. 5 and the employed hardware is listed in Table 1. Two accelerators were deployed on the spindle along the $X$ - and $Y$-axis directions of the CNC machine. The distance between the microphone and the cutter along the $Y$-axis is $85 \mathrm{~mm} .{ }^{(17)}$ 


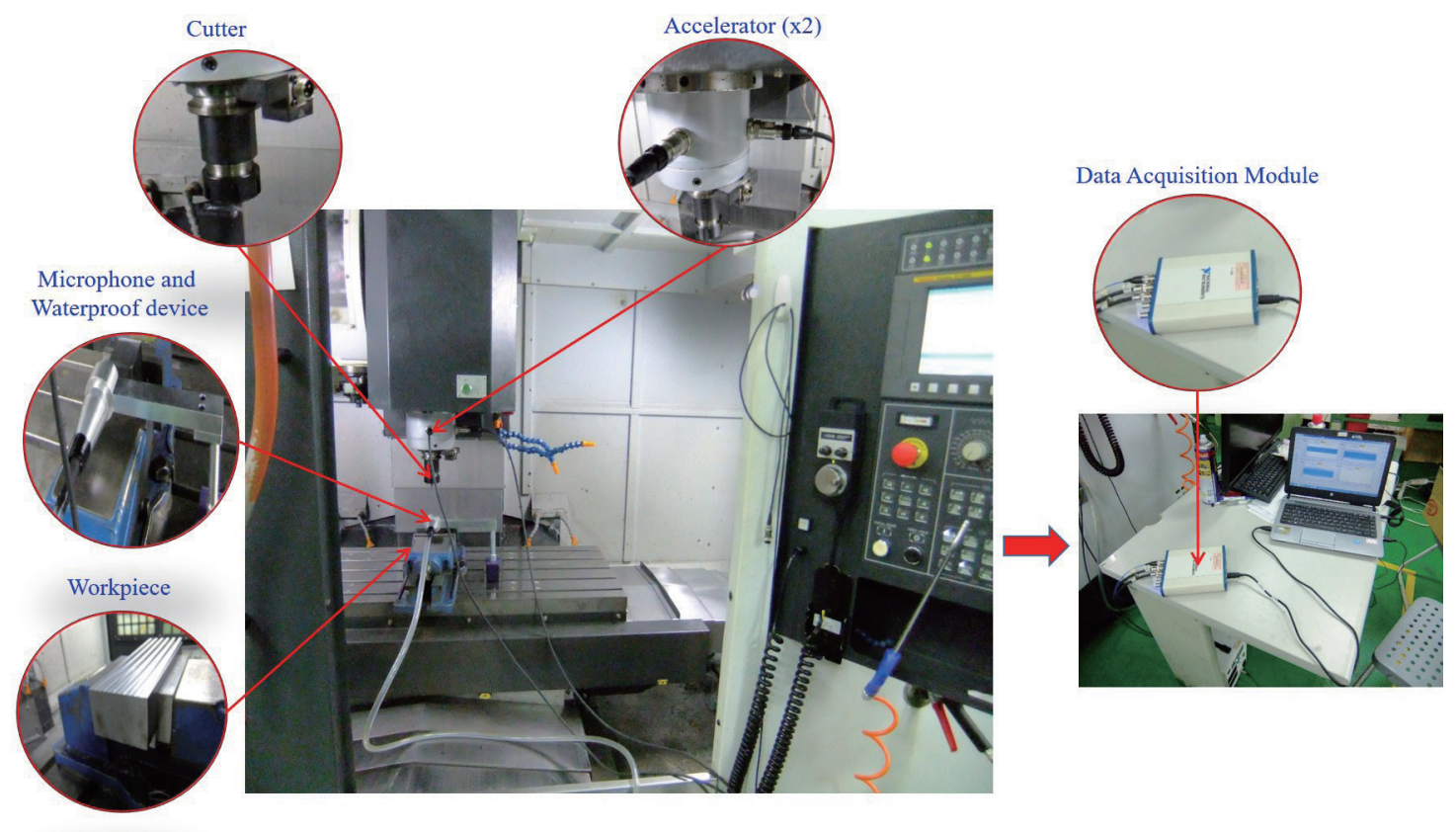

Fig. 5. (Color online) Test rig.

Table 1

Experimental hardware specifications.

\begin{tabular}{lcc}
\hline Item & Brand/Model & Remarks \\
\hline Microphone & PCB / 378C01 & Bandwidth: $20-80 \mathrm{kHz}$ \\
\hline Accelerometer & PCB / 601A01 & Bandwidth: $0.27-10 \mathrm{kHz}$ \\
\hline Laptop computer & HP / ProBook 430 G1 & For signal recording \\
\hline Data acquisition module & NI / USB-4431 & 4-port and USB interface \\
\hline CNC machine tool & FEELER / VMP-40A & Experimental machine \\
\hline Cutter & WEENIX / End mill & Material: cobalt high-speed steel \\
& & Number of teeth: 4 \\
Workpiece & & Diameter: $6 \mathrm{~mm}$ \\
\hline
\end{tabular}

\subsection{Cutting path design}

The SLD for the milling setup is shown in Fig. 6(a). For the purpose of inducing the chatter phenomenon, a bevel cutting path was specially designed as shown in Fig. 6(b). Since there are always certain degrees of errors inside the SLD, the maximum axial cut depth is set to be $6 \mathrm{~mm}$ to ensure the occurrence of chatter. There are six bevel cutting paths in one workpiece as shown in Figs. 6(c) and 6(d), three paths for dry cutting tests and the others for wet millings. In total, 12 millings were performed and the corresponding cutting parameters are listed in Table 2. 


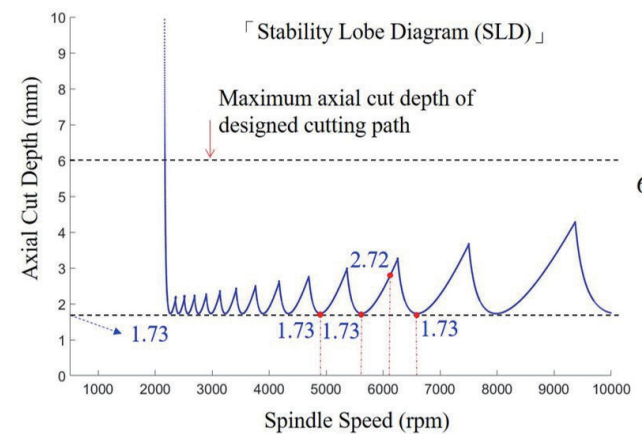

(a)

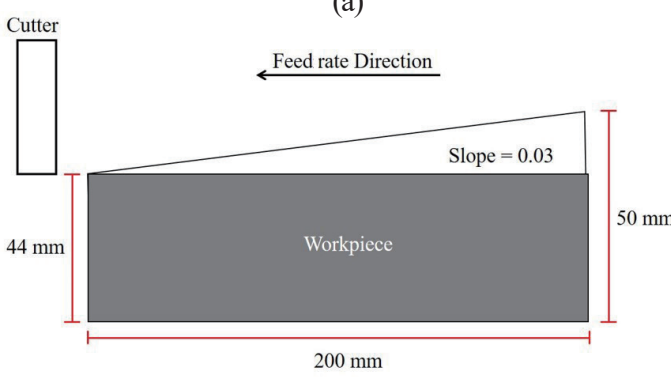

(c)

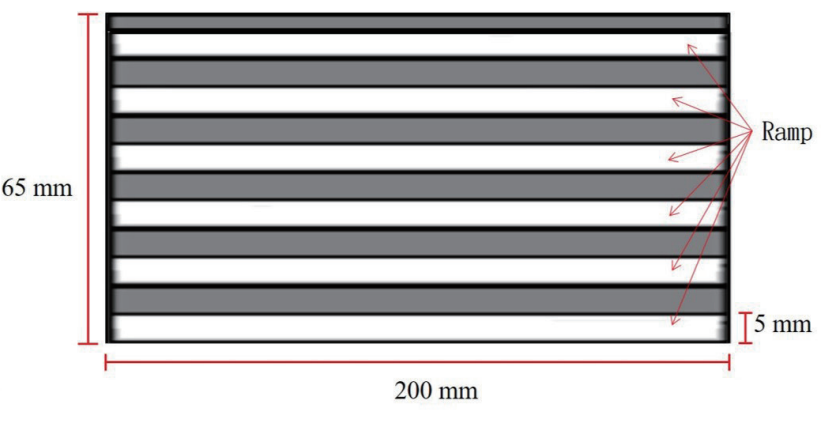

(b)

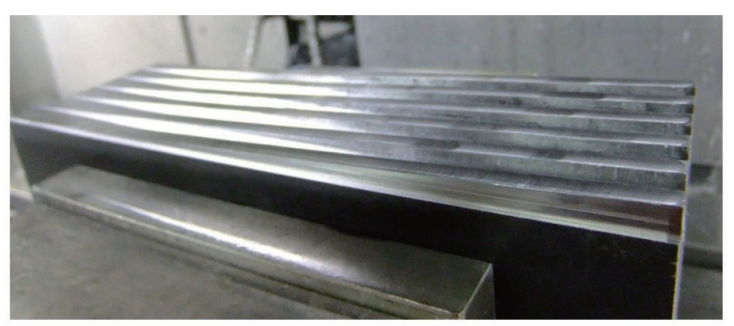

(d)

Fig. 6. (Color online) Cutting path design: (a) SLD for the experimental setup, (b) top view of workpiece design, (c) side view of workpiece design, and (d) practically prepared workpiece.

Table 2

Milling test parameters.

\begin{tabular}{lcccccc}
\hline Group & No. & $\begin{array}{c}\text { Spindle speed } \\
(\mathrm{rpm})\end{array}$ & $\begin{array}{c}\text { Feed rate } \\
(\mathrm{mm} / \mathrm{min})\end{array}$ & $\begin{array}{c}\text { Axial cut depth } \\
(\mathrm{mm})\end{array}$ & $\begin{array}{c}\text { Waterproof } \\
\text { device }\end{array}$ & Cutting fluid \\
\hline Stage A & A1 & 6600 & 2640 & $0-6(\mathrm{ramp})$ & Yes & No \\
Stage A & A2 & 6600 & 2640 & $0-6(\mathrm{ramp})$ & Yes & No \\
Stage A & A3 & 6600 & 2640 & $0-6(\mathrm{ramp})$ & Yes & No \\
Stage A & A4 & 6600 & 2640 & $0-6(\mathrm{ramp})$ & Yes & Yes \\
Stage A & A5 & 6600 & 2640 & $0-6(\mathrm{ramp})$ & Yes & Yes \\
Stage A & A6 & 6600 & 2640 & $0-6(\mathrm{ramp})$ & Yes & Yes \\
Stage B & B1 & 4900 & 1960 & $0-6(\mathrm{ramp})$ & Yes & No \\
Stage B & B2 & 4900 & 1960 & $0-6(\mathrm{ramp})$ & Yes & Yes \\
Stage B & B3 & 5600 & 2240 & $0-6(\mathrm{ramp})$ & Yes & No \\
Stage B & B4 & 5600 & 2240 & $0-6(\mathrm{ramp})$ & Yes & Yes \\
Stage B & B5 & 6100 & 2440 & $0-6(\mathrm{ramp})$ & Yes & No \\
Stage B & B6 & 6100 & 2440 & $0-6(\mathrm{ramp})$ & Yes & Yes \\
\hline
\end{tabular}

Remarks: Feed rate is determined on the basis of Ref. 20.

\subsection{Sensor signal processing and result comparison}

Accelerators were employed to be the comparison baseline of chatter detection. The accelerator output is processed by STFT to observe the tooth passing frequency (TPF) and its multiple frequencies. Once frequencies of high energy intensity other than TPFs are 
observed, the milling dynamics is considered to be unstable. In other words, these high-energy frequencies result from the unexpected cutting vibration. In Stage A, the STFT of accelerator output is utilized to affirm the time interval of the milling process, to check the occurrence of chatter, and to verify the detective capability of the microphone for the milling process. For the simplification of chatter occurrence detection, the CV of IF is utilized to define the diagnostic threshold for microphone signals. In Stage B, the diagnosis results using the CV threshold will be double-checked on the basis of STFT results of accelerator signals and the SLD of the test rig, where the accelerator is used to confirm the chatter occurrence and the SLD is used to estimate the approximate time of chatter occurrence.

\section{Experimental Results and Discussion}

The spindle speed for Stage A is $6600 \mathrm{rpm}$ so that the corresponding TPF is $440 \mathrm{~Hz}$. The STFT results of milling Nos. A1 (dry cutting) and A4 (wet cutting) are shown in Fig. 7. The TPFs, i.e., 440 and $880 \mathrm{~Hz}$, can be clearly noted, and the time interval of the milling process

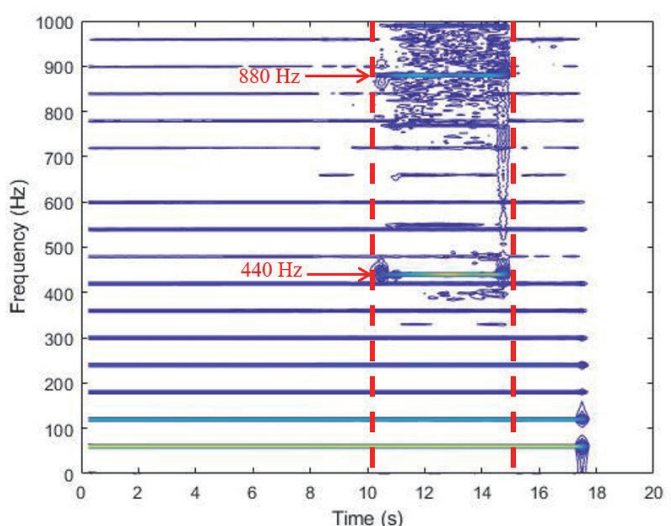

(a)

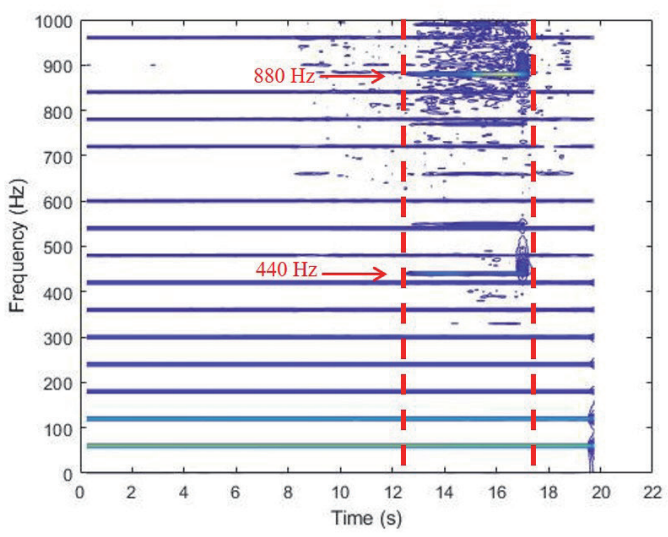

(c)

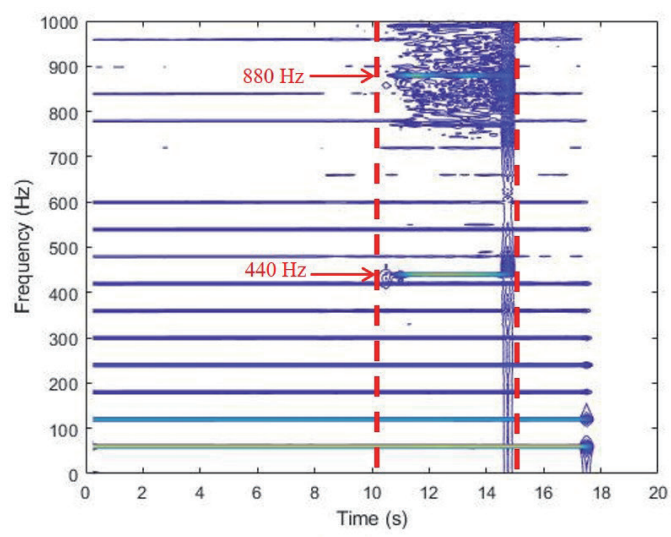

(b)

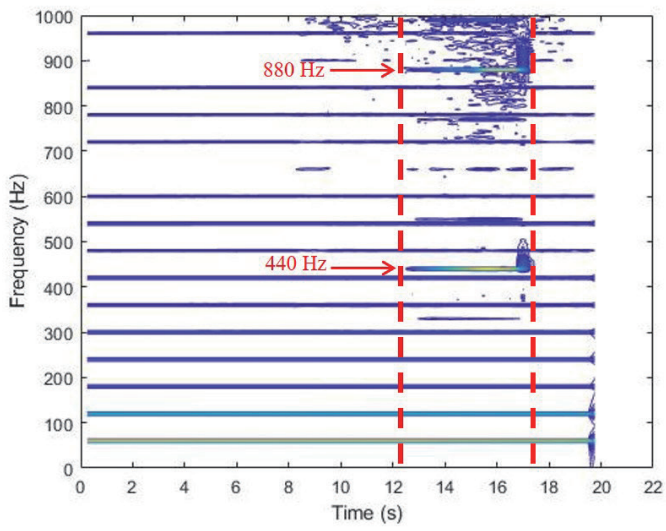

(d)

Fig. 7. (Color online) STFT of accelerator signals: (a) No. A1 in $X$-axis, (b) No. A1 in $Y$-axis, (c) No. A4 in $X$-axis, and (d) No. A4 in $Y$-axis. 
can be approximately estimated (between the two vertical dotted lines). The chaos in the upper parts of the STFT results refers to abnormal vibrations, e.g., chatter. Although the occurrence of chatter can be detected on the basis of the STFT results, the time point of the beginning of chatter can hardly be clarified. The microphone signals and the corresponding THT results of test Nos. A1 and A4 are shown in Fig. 8. The number marks in Figs. 8(b) and 8(d) are defined as follows:

zone 1: spindle motor starts

zone 2: spindle speed up

zone 3: spindle speed remains in the test speed without cutting

zone 4: initial phase of milling process

zone 5: chatter phase

zone 6: end phase of milling process (cutter just left the workpiece)

zone 7: spindle speed remains in the test speed without cutting

According to the cutting path design, the milling process includes zones 4 and 5. However, zones 4 to 6 should be the practical milling process owing to the errors of milling parameters and the milling complexity of the cutting path end. A thin wall was met in the end of the cutting path so that the practical material removal rate (MRR) can hardly be maintained, and therefore the milling dynamics becomes more complex. That is why zone 6 is also part of the milling process.

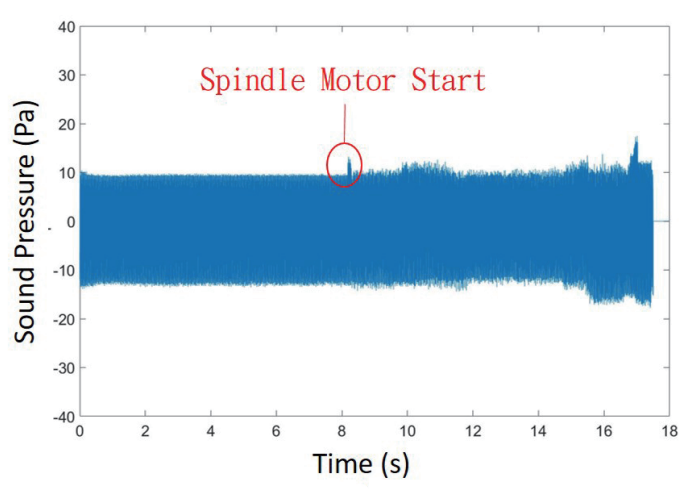

(a)

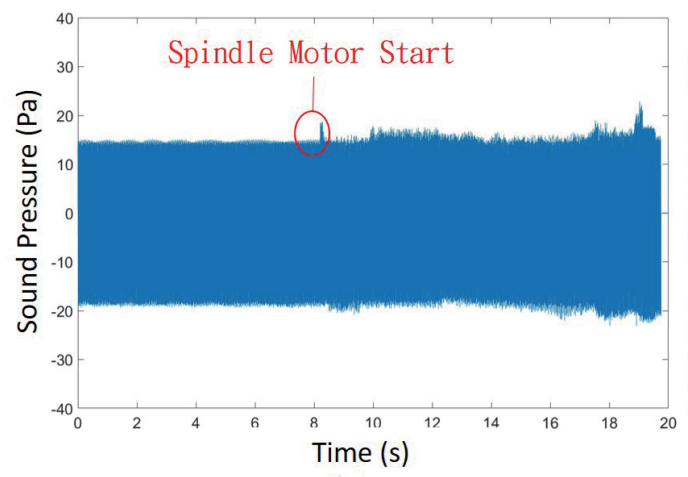

(c)

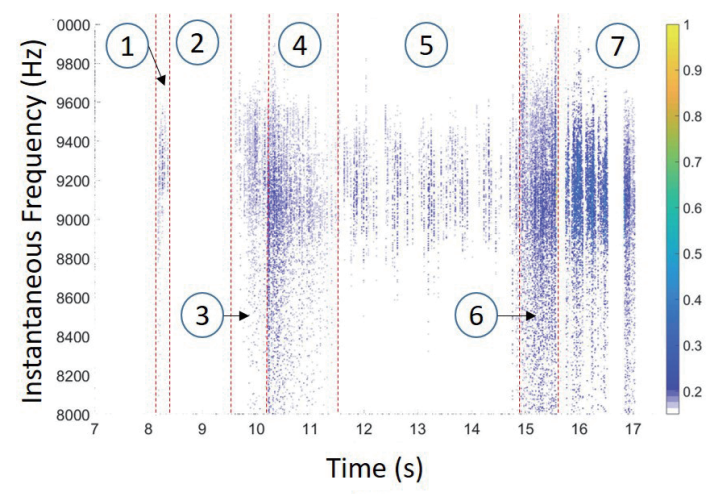

(b)

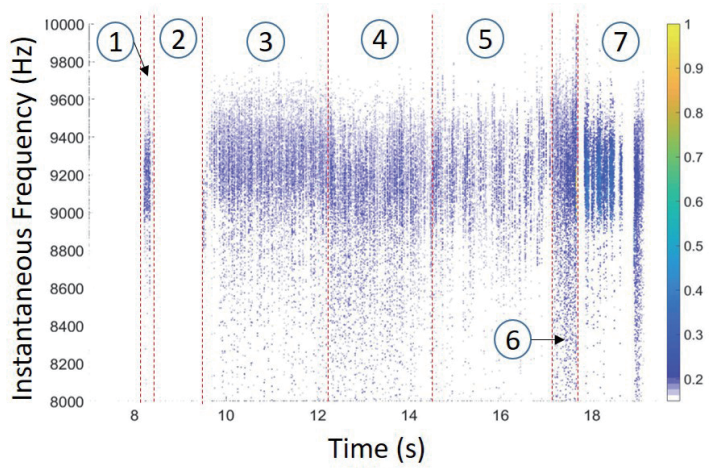

(d)

Fig. 8. (Color online) Microphone signals and THT results of tests: (a) and (b) No. A1, and (c) and (d) No. A4. 
The frequencies of the chatter phase for six tests (Nos. A1-A6) are mainly between 8800 and $9600 \mathrm{~Hz}$. In other words, the frequency range will decrease and the strength of certain frequencies will increase during the chatter phase. On the other hand, the time length of the chatter phase of wet milling is shorter than that of dry milling owing to the lubrication by the cutting fluid. The CVs of IF of THT results for six tests are shown in Fig. 9. On the basis of the $\mathrm{CV}$ results of six tests, the threshold of the CV for chatter detection is set as $13 \%$. The lower $\mathrm{CV}$ of IF refers to the lower dispersion degree of signal frequencies, i.e., the reduced frequency range.

Three groups of milling parameters were tested, i.e., test Nos. B1-B6, to verify the efficacy of the proposed method. The CVs of IF of THT results for test Nos. B1-B6 are shown in Fig. 10. The starting time point of the milling process was affirmed on the basis of the STFT
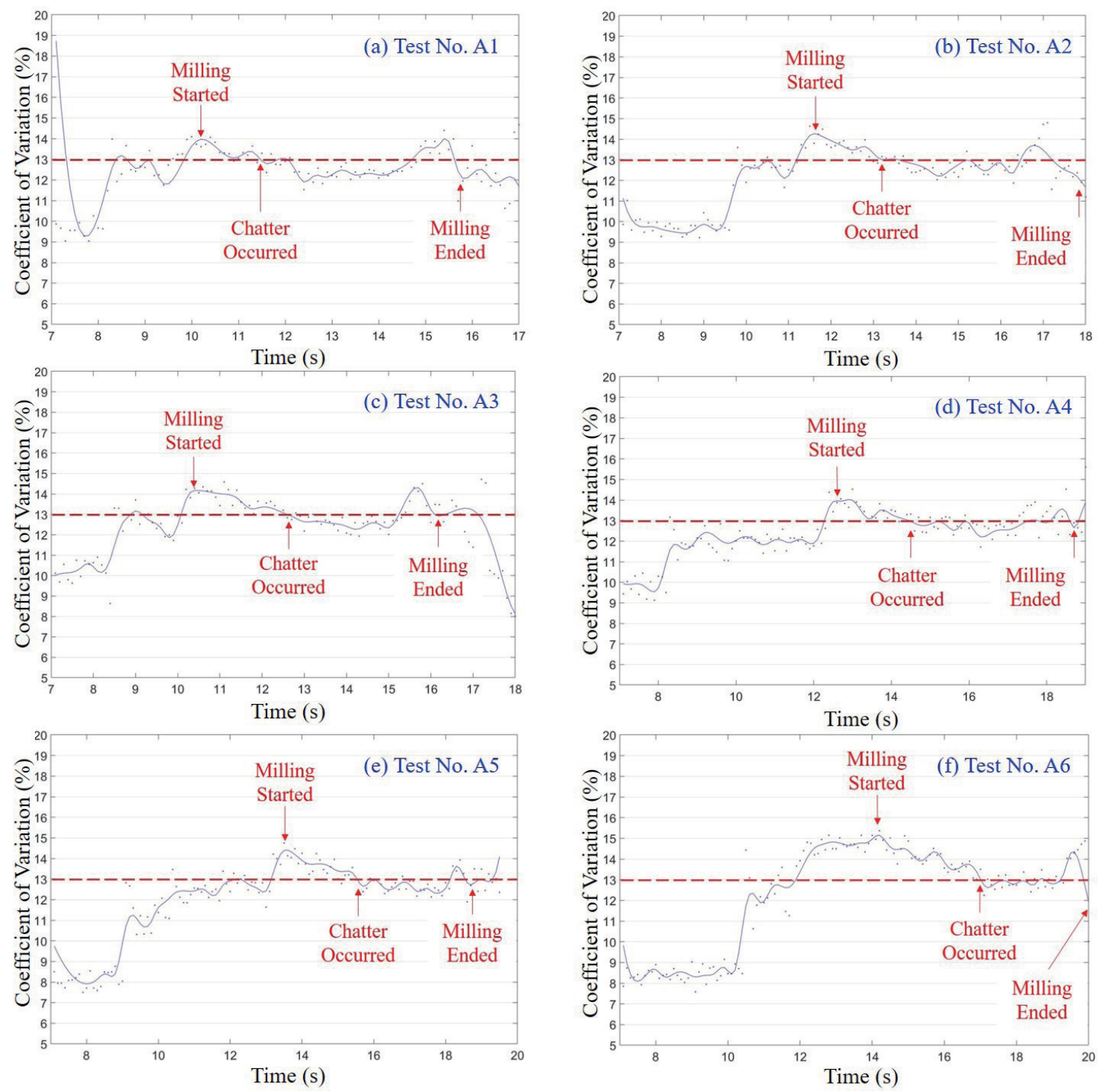

Fig. 9. (Color online) CVs of IF of THT results for test Nos. A1-A6. 

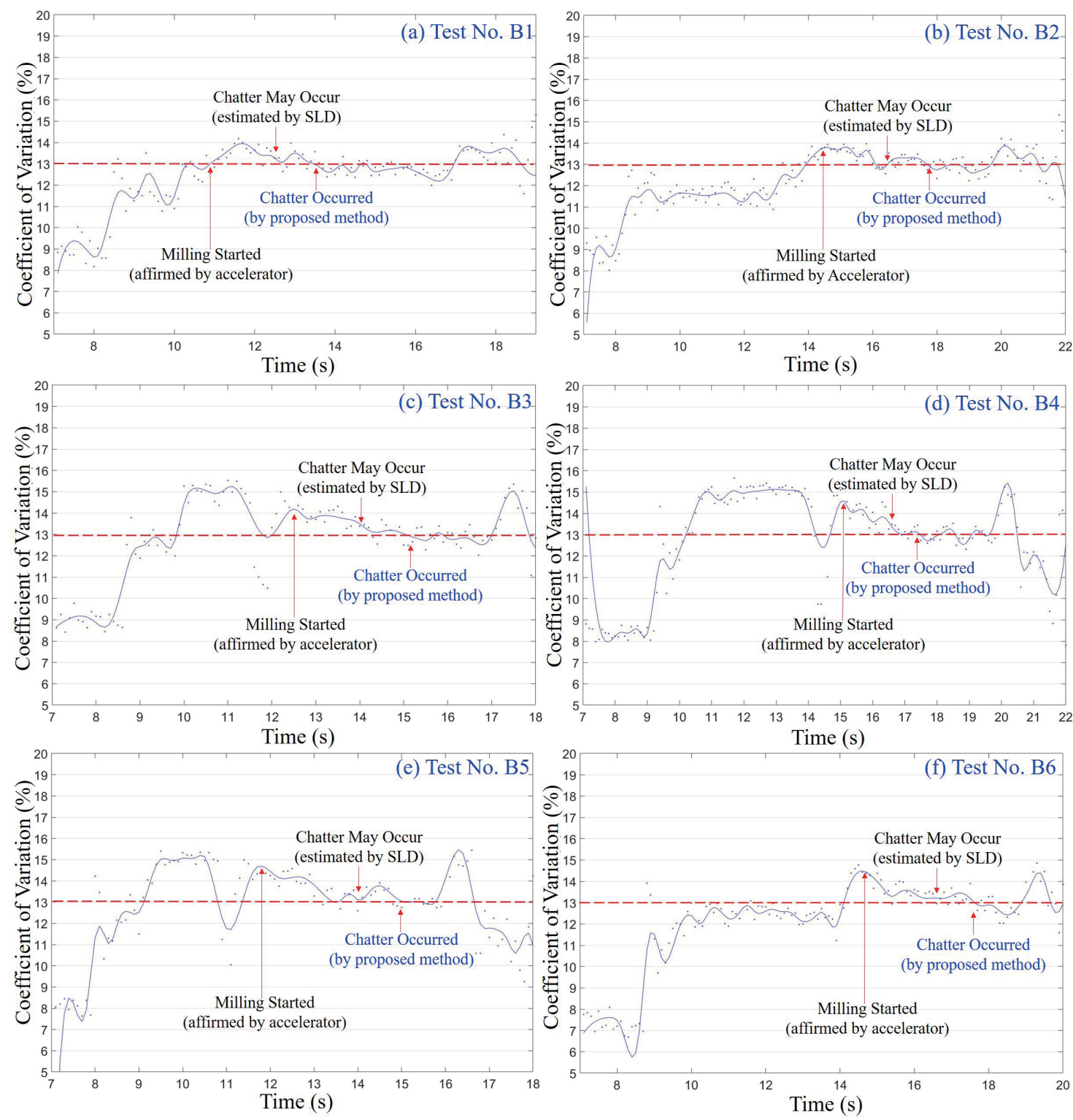

Fig. 10. (Color online) CVs of IF of THT results for test Nos. B1-B6.

results of accelerator signals, i.e., to observe the TPFs. The chatter phenomena in these six tests have also been confirmed using the accelerator, and the starting time point of the chatter phase was estimated on the basis of the SLD. For example, the critical axial cut depth is $2.72 \mathrm{~mm}$ for test Nos. B5 and B6 (see Fig. 6) and the given feedrate is $2440 \mathrm{~mm} / \mathrm{min}$. Therefore, the chatter may occur in $2.23 \mathrm{~s}$ after the milling starts. However, there are certain degrees of errors among the SLDs; thus, the occurrence of chatter should be estimated as a time interval instead of a time point. The efficacy of the proposed method for chatter detection based on the microphone signal has been practically confirmed. 


\section{Conclusions}

A microphone-signal-based chatter detection method has been proposed in this work. THT was employed to decompose the microphone signal into IF and IA. The variations of milling dynamics can be clearly observed in the energy-time-frequency representation by THT. For simplification, $\mathrm{CV}$ was employed to define the threshold for chatter detection, and the frequency range of $8-10 \mathrm{kHz}$ was selected for the microphone signal analysis to isolate the environmental noise and maintain the proper signal intensity at the same time. According to the results of practical cutting tests, the dispersion degrees of signal frequencies decreased during the chatter phase. A CV threshold of $13 \%$ was therefore chosen and verified by various milling tests. The proposed method is capable of chatter detection by a microphone regardless of dry or wet milling.

\section{Acknowledgments}

This research was supported by the Ministry of Science and Technology, Taiwan (under Grant No. MOST 108-2221-E-153-011) and National Pingtung University (under Grant No. NPTU-108-003).

\section{References}

1 M. Siddhpura and R. Paurobally: Int. J. Mach. Tools Manuf. 64 (2012) 27.

I. Grabec and P. Leskovar: Ultrason. 15 (1977) 17.

S. Y. Liang and D. A. Dorfeld: J. Eng. Ind. 111 (1989) 199.

4 R. Y. Chiou and S. Y. Liang: Int. J. Mach. Tools Manuf. 40 (2000) 927.

5 I. Marinescu and D. Axinte: Int. J. Mach. Tools Manuf. 49 (2009) 53.

6 N. Grossi, A. Scippa, L. Sallese, R. Sato, and G. Campatelli: Int. J. Mach. Tools Manuf. 89 (2015) 221.

7 M. Lamraoui, M. Barakat, M. Thomas, and M. E. Badaoui: J. Vib. Control 21 (2013) 1251.

8 Z. Han, H. Jin, D. Han, and H. Fu: Int. J. Adv. Manuf. Technol. 89 (2017) 2731.

9 B. Cuka, M. Cho, and D.-W. Kim: Procedia Manuf. 17 (2018) 142.

10 M. Lu, B. Chen, D. Zhao, J. Zhou, J. Lin, A. Yi, and H. Wang: Appl. Sci. 9 (2019) 21.

11 Z. Yao, D. Mei, and Z. Chen: J. Mater. Process. Technol. 210 (2010) 713.

12 G. Wang, H. Dong, Y. Guo, and Y. Ke: Int. J. Adv. Manuf. Technol. 94 (2018) 1243.

13 M. Uekita and Y. Takaya: Meas. 103 (2017) 199.

14 T. Thaler, P. Potocnik, I. Bric, and E. Govekar: Appl. Acoust. 77 (2014) 114.

15 J.-C. Cexus and A.-O. Boudraa: 14th Eur. Signal Process. Conf. (2006) 15033449.

16 H. Li, H. Zheng, and L. Tang: Int. J. Rotating Mach. 2010 (2010) 1.

17 R.-M. Lee, P.-T. Liu, and C.-C. Wang: Sens. Mater. 30 (2018) 2419.

18 N. E. Huang, Z. Shen, S. R. Long, M. C. Wu, H. H. Shih, Q. Zheng, N.-C. Yen, C. C. Tung, and H. H. Liu: Proc. R. Soc. London, Ser. A 454 (1998) 903.

19 P. Maragos, J. F. Kaiser, and T. F. Quatieri: IEEE Trans. Signal Process. 41 (1993) 3024.

20 S.-C. J. Lin: Computer Numerical Control: From Programming to Networking (Delmar Cengage Learning, New York, 1994). 